\title{
Tetrasomy 9p Mosaicism Associated with a Normal Phenotype in Two Cases
}

\author{
I. Papoulidis ${ }^{a} \quad$ M. Kontodiou ${ }^{a} \quad$ M. Tzimina ${ }^{a} \quad$ I. Saitis ${ }^{b} \quad$ A.B. Hamid ${ }^{c} \quad$ E. Klein ${ }^{c}$

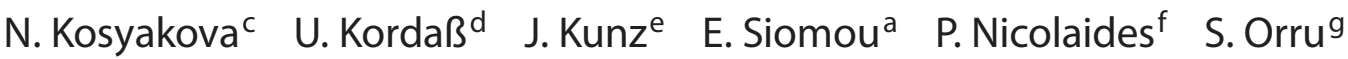 \\ L. Thomaidis ${ }^{\mathrm{h}} \quad$ T. Liehr ${ }^{\mathrm{c}} \quad$ M.B. Petersen ${ }^{\mathrm{a}} \quad$ E. Manolakos ${ }^{\mathrm{a}, \mathrm{g}}$ \\ ${ }^{a}$ Eurogenetica S.A., Thessaloniki and Athens, ${ }^{b}$ Genemed, Heraklion, Greece; ${ }^{C}$ Institute of Human Genetics, \\ Jena University Hospital, Jena, 'Institute of Human Genetics, Greifswald University Hospital, Greifswald, \\ e Institute of Human Genetics, Charité, Berlin, Germany; ${ }^{\mathrm{f}}$ Emvrioiatriki S.A., Athens, Greece; ${ }^{9}$ Cattedra di \\ Genetica Medica, Ospedale Binaghi, Universita di Cagliari, Cagliari, Italy; ${ }^{\text {h} D e v e l o p m e n t a l ~ A s s e s s m e n t ~ U n i t, ~}$ \\ 2nd Department of Pediatrics, P. \& A. Kyriakou Children's Hospital, National and Kapodistrian University of \\ Athens School of Medicine, Athens, Greece
}

\section{Key Words}

Mosaicism $\cdot$ Normal phenotype $\cdot$ Tetrasomy 9p

\begin{abstract}
Tetrasomy $9 p$ is a rare chromosomal syndrome and about $30 \%$ of known cases exhibit mosaicism. Approximately 50 of the reported cases with tetrasomy $9 p$ mosaicism show a characteristic facial appearance, growth failure, and developmental delay. However, 3 patients with mosaicism for isochromosome $9 p$ and a normal phenotype have also been reported. We report 2 additional cases of clinically normal young females with tetrasomy $9 p$ mosaicism, one of whom also exhibited $X$ chromosome aneuploidy mosaicism leading to an overall of 6 different cell lines. STR analysis performed on this complex mosaic case indicated that the extra isochromosome was of maternal origin while the $\mathrm{X}$ chromosome aneuploidy was of paternal origin, indicating a postzygotic event.

Copyright $\odot 2012$ S. Karger AG, Basel
\end{abstract}

Small supernumerary marker chromosomes (sSMCs) are reported in $0.044 \%$ of newborn infants and in $0.125 \%$ of subfertile individuals [Liehr and Weise, 2007]. sSMCs are structurally abnormal chromosomes that cannot be identified or characterized unambiguously by conventional cytogenetics alone, and they are generally equal in size or smaller than chromosome 20 of the same metaphase spread [Liehr et al., 2004]. To date, only one report is available for a triple-X syndrome patient with an additional sSMC [Lee-Jones et al., 2004], and only 3 cases have been reported so far with mosaic tetrasomy $9 p$ that present no clinical symptoms [Sait and Wetzler, 2003; McAuliffe et al., 2005; Baronchelli et al., 2011].

In the present study, we report 2 patients with tetrasomy $9 \mathrm{p}$ mosaicism and an apparently normal phenotype. The first individual was cytogenetically studied because of a de novo inversion in a chromosome 7, observed in a previous pregnancy. The second proband was referred for cytogenetic studies as part of in vitro fertilization (IVF) pre-testing due to her husband's azoospermia. The results of the molecular, clinical, and cytogenetic findings are presented and compared to reports previously published. 




Fig. 1. a Partial karyogram of case 1 showing both normal chromosomes 9 and the isochromosome 9 p in inverted DAPI-banding and after FISH. An alpha-satellite-specific probe for chromosome 9 (cep 9) and a subtelomeric probe for 9pter (subtel 9pter) were applied. b Both normal chromosomes 9 and the isochromosome 9p found in case 2 after FISH using an alpha-satellite-specific probe for chromosome 9 (cep 9) together with a microdissectionderived probe (midi36) for the pericentric region of chromosome $9(9 \mathrm{p} 12 / 9 \mathrm{q} 13-21.1)$ and a whole chromosome painting (wcp) probe.

\section{Case Reports}

Case 1

A 20-year-old female was studied cytogenetically due to a previous pregnancy with a de novo pericentric inversion in a chromosome 7. The patient presented no dysmorphic features and/or mental abnormalities, and there was no family history of miscarriages and/or genetic abnormalities. Peripheral blood and buccal mucosa were available for cytogenetic studies.

\section{Case 2}

A 28-year-old female, the second child of healthy non-consanguineous parents, was studied cytogenetically before starting IVF treatment due to her husband's azoospermia. The family history was unremarkable. The patient had a height of $169 \mathrm{~cm}$, head circumference of $55 \mathrm{~cm}$, weight $63 \mathrm{~kg}$, had normally developed genitalia, normal menstrual cycle, and an average mental condition. Endocrinological studies revealed no abnormal values (PRL 23.3 ng/ml, FSH $9.7 \mathrm{mIU} / \mathrm{ml}, \mathrm{LH} 3.3 \mathrm{mIU} / \mathrm{ml}, \mathrm{E}_{2} 74.7 \mathrm{pg} / \mathrm{ml}$, PRG 0.41 ng/ml, 17-OH PRG 0.53 ng/ml, and DHEA 946.4 ng/ml).

\section{Methods and Results}

Metaphase chromosome preparations were obtained from PHA-stimulated lymphocyte cultures according to standard procedures [Verma and Babu, 1998].

In case 1, the cytogenetic analysis of stimulated blood cells revealed a non-mosaic karyotype of $47, \mathrm{XX},+$ mar. Multiplex-fluorescence in situ hybridization (M-FISH) [Speicher et al., 1996] showed that the sSMC was a derivative of chromosome 9. Application of a centromeric probe for chromosome 9 (cep 9) in combination with a subtelomeric probe for the short arm of chromosome 9 (9pter) identified the sSMC as an i(9)(p10) (fig. 1a). However, in buccal mucosa, interphase-FISH, using a centromeric probe for chromosome 9, confirmed the presence of the sSMC only in $65 \%$ of the examined cells. This finding in association with the normal clinical phenotype of the patient indicates that it is possible that most of the tissues of the patient present a mosaicism for isochromosome $9 \mathrm{p}$ rather than a full tetrasomy $9 \mathrm{p}$. According to ISCN [2009], the karyotype was mos 47,XX,+i(9)(p10)/ $46, \mathrm{XX}$. Follow-up cytogenetic studies of the patient's parents were not possible.

In case 2 , a routine cytogenetic analysis on peripheral blood revealed a mosaic karyotype mos 48,XXX,+mar [14]/47,XX,+mar[14]/49,XXXX,+mar[4]/47,XXX[2]/46,X, $+\operatorname{mar}[2] / 46, \mathrm{XX}[4]$. Parental chromosome analysis revealed normal karyotypes. Application of an alpha-satellite-specific probe for chromosome 9 (cep 9) together with a microdissection-derived probe (midi36) for the pericentric region of chromosome 9 (9p12/9q13-21.1), and a whole chromosome painting probe identified the sSMC as an i(9)(p10) (fig. 1b). Accordingly, the karyotype was designated as mos 48, XXX,+i(9)(p10)[14]/47, XX,+i(9) (p10)[14]/49,XXXX,+i(9)(p10)[4]/47,XXX[2]/46,X,+i(9) (p10)[2]/46,XX[4]dn.

DNA was extracted from blood samples using the $\mathrm{Nu}-$ cleoSpin blood extraction kit (Macherey-Nagel, Düren, Germany). Uniparental disomy (UPD) of the normal chromosomes 9 was excluded by means of parent-to-patient segregation analysis using a panel of 8 short tandem repeat (STR) markers located along the length of chromosome 9 (D9S103, D9S117, D9S199, D9S194, D9S195, D9S109, D9S193, D9S200). A set of 4 STR markers was also used for the determination of the origin of the $\mathrm{X}$ chromosome aneuploidy (DXS990, DXS987, DXS8091, DXS1047). Quantitative fluorescence (QF) PCR was performed to amplify the repeat sequences at the above polymorphic loci, and the primer sequences were probed with fluorescent labels as described elsewhere [Mann et al., 
Table 1. Cytogenetic findings and clinical data in 4 mosaic cases with a supernumerary i(9p)

\begin{tabular}{|c|c|c|c|c|c|}
\hline & $\begin{array}{l}\text { McAuliffe } \\
\text { et al., } 2005\end{array}$ & Sait and Wetzler, 2003 & $\begin{array}{l}\text { Baronchelli } \\
\text { et al., } 2011\end{array}$ & Case 1 & Case 2 \\
\hline Age, years & 37 & 41 & adult & 20 & 28 \\
\hline Sex & male & male & female & female & female \\
\hline Phenotype & normal & normal/skin lesions/hypereosinophilia & normal & normal & normal \\
\hline $\begin{array}{l}\text { Reason for } \\
\text { investigation }\end{array}$ & oligospermia & $\begin{array}{l}\text { hypereosinophilia in bone marrow } \\
\text { and peripheral blood film/skin lesions }\end{array}$ & $\begin{array}{l}\text { premature ovarian } \\
\text { failure }\end{array}$ & previous pregnancy inv(7) & IVF \\
\hline Origin & n.a. & n.a. & n.a. & n.a. & de novo \\
\hline
\end{tabular}

$\mathrm{PBL}=$ Peripheral blood lymphocytes.

2001]. The fluorescent QF-PCR products were analyzed by capillary electrophoresis on an automated DNA sequencer (ABI 3100, Applied Biosystems, Carlsbad, Calif., USA). STR analysis indicated that the extra isochromosome was of maternal origin, while the $\mathrm{X}$ chromosome aneuploidy observed in case 2 was of paternal origin (all extra copies of chromosome X).

STR analysis was not performed in case 1 as no parental DNA material was available.

\section{Discussion}

Tetrasomy $9 \mathrm{p}$ is a rare syndrome, and about $30 \%$ of known cases exhibit chromosome mosaicism [Stumm et al., 1999]. Reports in the literature of cases with tetrasomy 9p, about 50 including mosaic and non-mosaic cases [Liehr, 2011], showed characteristic facial appearance with hypertelorism (72\%), broad nasal root or bulbus/ beaked nose (69\%), cleft lip or palate (78\%), ear anomalies (88\%), micrognathia (59\%), developmental delay (94\%), central nervous system anomaly (89\%), limb defects (88\%), postnatal growth failure (71\%), congenital heart disease (62\%), small gestational age (57\%), renal anomalies (57\%), wide sutures/large fontanelle (56\%), and short neck/excess nuchal skin (53\%) [Dhandha et al., 2002].

There are 3 patients depicted in the literature with mosaicism for isochromosome $9 \mathrm{p}$ and a normal phenotype
[Sait and Wetzler, 2003; McAuliffe et al., 2005; Baronchelli et al., 2011] (table 1). Sait and Wetzler [2003] described a healthy 41 -year-old male with mosaicism of isochromosome $9 \mathrm{p}$ who was referred for cytogenetic analysis because of skin lesions; the only abnormality found was hypereosinophilia in the bone marrow and peripheral blood film. McAuliffe et al. [2005] reported a 37-yearold male patient with isochromosome 9p mosaicism with oligospermia who had fathered 2 normal children, and Baronchelli et al. [2011] found an i $(9 p)$ in $72 \%$ of peripheral blood cells studied cytogenetically in an adult female with premature ovarian failure.

In the 2 additional cases reported here, the chromosomal imbalance of chromosome 9 was not associated with any prominent phenotypic abnormality in the apparently healthy 20- and 28-year-old females. It has been proposed that the degree of phenotypic involvement can be associated with the degree of mosaicism, the size of the isochromosome involved, and the extent of tissue involvement [Grass et al., 1993].

Although a correlation between the level of mosaicism and phenotypic abnormalities has been described, there was no such evidence in our 2 cases. Interestingly, similar findings have been reported for other sSMC cases usually known to have an adverse prognosis but instead presented a mild phenotype, such as additional isochromosome 18p [Kim et al., 2009], inv dup(15)(q13) [Bonati et al., 2005; Loitzsch and Bartsch, 2006], inv dup(22)(q11.21) 
leading generally to cat eye syndrome [Lin et al., 2006], or even isochromosome 12 p leading to Pallister-Killian syndrome [Genevieve et al., 2003]. In our case 1, no mosaicism was evident after studying blood lymphocytes; it became obvious only after interphase-FISH of the buccal mucosa. Still, only a few tissues were studied in both of our cases.

The supernumerary isochromosome $9 \mathrm{p}$ in case 2 was a de novo finding as in all of the so far described cases [Dutly et al., 1998; Eggerman et al., 1998; Wyandt et al., 2000] and of maternal origin. It seems that errors in maternal meiosis may be responsible for the origin of the isochromosome and that non-disjunction during meiosis II could be followed by rearrangements leading to duplication of the short arm and loss of the long arm in the majority of cases [Dutly et al., 1998].

For sSMCs in general, the predominant mechanism of origin has been shown to be ring chromosome formation by centromere misdivision, the so-called McClintock mechanism [Baldwin et al., 2008].

Trisomy $\mathrm{X}$ occurs from a non-disjunction event in which the X chromosomes fail to properly separate during cell division, either during gametogenesis or after conception [May et al., 1990]. Studies made to determine the parental origin of the additional X chromosome demonstrated that in $58-63 \%$ of cases the extra X chromosome derived from maternal meiosis I errors, in $16-17 \%$ from maternal meiosis II errors, and in $18-20 \%$ from post-zygotic non-disjunction [Hall et al., 2006; Hassold et al., 2007]. One study [Wallerstein et al., 2004] with mo- saic trisomy $\mathrm{X}$ (such as $45, \mathrm{X} / 47, \mathrm{XXX}$ ) suggested that cases of mosaicism may result from a post-zygotic non-disjunction event as could be the cause in our case 2 . This case presented with a normal stature, while women with a mosaic karyotype of 45,X/47,XXX generally develop a short stature [Syber and McCauley, 2004]. The severity of the short stature has been correlated with the distribution of cell lines in 47,XXX/45,X/46,XX mosaicism [Partsch et al., 1994].

Mosaicism for tetrasomy $9 \mathrm{p}$ is a challenging issue in terms of prenatal diagnosis and genetic counseling as the abnormality may not be detectable in the amniotic fluid and fetal ultrasound assessment can be normal throughout pregnancy. In one reported case, amniocentesis due to advanced maternal age showed a normal fetal karyotype. However, further cytogenetic analysis due to postnatal developmental delay revealed mosaic tetrasomy $9 p$ in blood and skin cells [Eggermann et al., 1998].

Our 2 cases of healthy females can be regarded as representing the one end of the spectrum of karyotype-phenotype correlation in chromosomal aneuploidies [Avramopoulos et al., 1997]. In most such cases, however, tissue-specific mosaicism has not been fully investigated.

\section{Acknowledgments}

This article was supported in parts by the Else-Kröner-Fresenius Stiftung and the DAAD.

\section{References}

Avramopoulos D, Kennerknecht I, Barbi G, Eckert D, Delabar JM, et al: A case of apparent trisomy 21 without the Down's syndrome phenotype. J Med Genet 34:597-600 (1997).

Baldwin EL, May LF, Justice AN, Martin CL, Ledbetter DH: Mechanisms and consequences of small supernumerary marker chromosomes: from Barbara McClintock to modern genetic-counselling issues. Am J Hum Genet 82:398-410 (2008).

Baronchelli S, Conconi D, Panzeri E, Bentivegna A, Redaelli S, et al: Cytogenetics of premature ovarian failure: an investigation on 269 affected women. J Biomed Biotechnol 2011: 370195 (2011).

Bonati MT, Finelli P, Giardino D, Gottardi G, Roberts W, Larizza L: Trisomy 15q25.2-qter in an autistic child: genotype-phenotype correlations. Am J Med Genet A 133:184-188 (2005).
Dhandha S, Hogge WA, Surti U, McPherson E: Three cases of tetrasomy 9p. Am J Med Genet 113:375-380 (2002).

Dutly F, Balmer D, Baumer A, Binkert F, Schinzel A: Isochromosomes 12p and 9p: parental origin and possible mechanisms of formation. Eur J Hum Genet 6:140-144 (1998).

Eggermann T, Rossier E, Theurer-Mainka U, Backsch C, Klein-Vogler U, et al: New case of mosaic tetrasomy 9p with additional neurometabolic findings. Am J Med Genet 75:530533 (1998).

Genevieve D, Cormier-Daire V, Sanlaville D, Faivre L, Gosset P, et al: Mild phenotype in a 15 -year-old boy with Pallister-Killian syndrome. Am J Med Genet 116A:90-93 (2003).

Grass FS, Parke JC Jr, Kirkman HN, Christensen $\mathrm{V}$, Roddey OF, et al: Tetrasomy 9p: tissuelimited idic(9p) in a child with mild manifestations and a normal CVS result. Report and review. Am J Med Genet 47:812-816 (1993).
Hall H, Hunt P, Hassold T: Meiosis and sex chromosome aneuploidy: how meiotic errors cause aneuploidy; how aneuploidy causes meiotic errors. Curr Opin Genet Dev 16: 323-329 (2006).

Hassold T, Hall H, Hunt P: The origin of human aneuploidy: where we have been, where we are going. Hum Mol Genet $16 \mathrm{Spec}$ No. 2:R203-208 (2007).

ISCN 2009: An International System for Human Cytogenetic Nomenclature (200p), Shaffer LG, Slovak ML, Campbel LJ (eds) (S. Karger, Basel 2009).

Kim M, Park C, Park S, Kim M, Lee B, et al: Prenatal diagnosis of a de novo mosaic isochromosome 18p: karyotype discordance between amniocytes and fetal/neonatal blood. ASHG 2009; abstract only online, information from poster. 
Lee-Jones L, Williams T, Little E, Sampson J: Trisomy 14 pter $\rightarrow$ q21: a case with associated ovarian germ cell tumor and review of the literature. Am J Med Genet A 128A:78-84 (2004).

Liehr T: Small supernumerary marker chromosomes. http://www.fish.uniklinikum-jena. de/sSMC.html. [accessed 20/02/2012].

Liehr T, Weise A: Frequency of small supernumerary marker chromosomes in prenatal, newborn, developmentally retarded and infertility diagnostics. Int J Mol Med 19:719731 (2007).

Liehr T, Claussen U, Starke H: Small supernumerary marker chromosomes (sSMC) in humans. Cytogenet Genome Res 107:55-67 (2004).

- Lin CC, Hsieh YY, Wang CH, Li YC, Hsieh LJ, et al: Prenatal detection and characterization of a small supernumerary marker chromosome (sSMC) derived from chromosome 22 with apparently normal phenotype. Prenat Diagn 26:898-902 (2006).

Loitzsch A, Bartsch O: Healthy 12-year-old boy with mosaic inv dup(15)(q13). Am J Med Genet A 140:640-643 (2006).

-Mann K, Fox SP, Abbs SJ, Yau SC, Scriven PN, et al: Development and implementation of a new rapid aneuploidy diagnostic service within the UK National Health Service and implications for the future of prenatal diagnosis. Lancet 358:1057-1061 (2001).
May KM, Jacobs PA, Lee M, Ratcliffe S, Robinson $A$, et al: The parental origin of the extra $\mathrm{X}$ chromosome in 47,XXX females. Am J Hum Genet 46:754-761 (1990).

-McAuliffe F, Winsor EJ, Chitayat D: Tetrasomy $9 \mathrm{p}$ mosaicism associated with a normal phenotype. Fetal Diagn Ther 20:219-222 (2005).

Partsch CJ, Pankau R, Sippell WG, Tolksdorf M: Normal growth and normalization of hypergonadotropic hypogonadism in atypical Turner syndrome $(45, \mathrm{X} / 46, \mathrm{XX} / 47, \mathrm{XXX})$. Correlation of body height with distribution of cell lines. Eur J Pediatr 153:451-455 (1994).

Sait SNJ, Wetzler M: Tetrasomy 9p with no apparent phenotype characteristics (abstract), 53th ASHG Annual Meeting: Nr 678 (2003).

Speicher MR, Gwyn Ballard S, Ward DC: Karyotyping human chromosomes by combinatorial multi-fluor FISH. Nat Genet 12:368-375 (1996).

-Stumm M, Tönnies H, Mandon U, Götze A, Krebs P, Wieacker PF: Mosaic tetrasomy 9p in a girl with multiple congenital anomalies: cytogenetic and molecular-cytogenetic studies. Eur J Pediatr 158:571-575 (1999).

Syber VR, McCauley E: Turner's syndrome. N Engl J Med 351:1227-1238 (2004).

Verma RS, Babu A: Human Chromosomes Manual of Basis Technologies, ed 4, pp 6-71 (Pergamon Press, New York 1998).
Wallerstein R, Musen E, McCarrier J, Aisenberg J, Chartoff A, et al: Turner syndrome phenotype with 47,XXX karyotype: further investigation warranted? Am J Med Genet A 125A:106-107 (2004).

Wyandt HE, Lebo RV, Fenerci EY, Sadhu DN, Milunsky JM: Tandem duplication/deletion in a maternally derived chromosome 9 supernumerary derivative resulting in $9 p$ trisomy and partial 9q tetrasomy. Am J Med Genet 93:305-312 (2000).

\section{Notes Added in Proof}

A more intense literature search revealed four (4) additional i(9p) cases with normal phenotype. For more details visit: http://www.fish.uniklinikum-jena.de/ sSMC/sSMC+by+chromosome/sSMC+9. html\#i9p). 